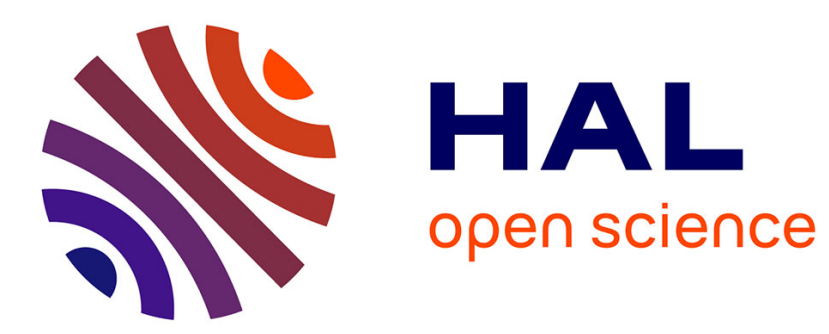

\title{
ExoY, an actin-activated nucleotidyl cyclase toxin from $P$. aeruginosa: a minireview
}

Alexander Belyy, Undine Mechold, Louis Renault, Daniel Ladant

\section{To cite this version:}

Alexander Belyy, Undine Mechold, Louis Renault, Daniel Ladant. ExoY, an actinactivated nucleotidyl cyclase toxin from P. aeruginosa: a minireview. Toxicon, 2017, 10.1016/j.toxicon.2017.12.046 . pasteur-01765203

\section{HAL Id: pasteur-01765203}

\section{https://hal-pasteur.archives-ouvertes.fr/pasteur-01765203}

Submitted on 12 Apr 2018

HAL is a multi-disciplinary open access archive for the deposit and dissemination of scientific research documents, whether they are published or not. The documents may come from teaching and research institutions in France or abroad, or from public or private research centers.
L'archive ouverte pluridisciplinaire HAL, est destinée au dépôt et à la diffusion de documents scientifiques de niveau recherche, publiés ou non, émanant des établissements d'enseignement et de recherche français ou étrangers, des laboratoires publics ou privés. 
1 ExoY, an actin-activated nucleotidyl cyclase toxin from $P$. aeruginosa: a

\section{2 minireview}

$4 \quad$ Alexander Belyy ${ }^{1}$, Undine Mechold ${ }^{1}$, Louis Renault ${ }^{2}$ and Daniel Ladant ${ }^{1}$.

5

61 - Institut Pasteur, CNRS UMR3528, Unité de Biochimie des Interactions 7 Macromoléculaires, Département de Biologie Structurale et Chimie, 25-28 rue du 8 Docteur Roux, 75724 Paris cedex 15, France.

92 - Institute for Integrative Biology of the Cell (I2BC), CEA, CNRS, Univ. Paris-Sud, 10 Université Paris-Saclay, 91198 Gif-sur-Yvette cedex, France.

\section{Abstract}

13 ExoY is one of four well-characterised Pseudomonas aeruginosa type 3 secretion system (T3SS) effectors. It is a nucleotidyl cyclase toxin that is inactive inside the bacteria, but becomes potently activated once it is delivered into the eukaryotic target cells. Recently, filamentous actin was identified as the eukaryotic cofactor that stimulates specifically ExoY enzymatic activity by several orders of magnitude. In this review, we discuss recent advances in understanding the biochemistry of nucleotidyl cyclase activity of ExoY and its regulation by interaction with filamentous actin.

\section{Keywords}

Bacterial protein toxin; ExoY; Pseudomonas aeruginosa; T3SS, actin.

\section{Highlights}

25 ExoY is an effector of the opportunistic human pathogen Pseudomonas aeruginosa. 
ExoY belongs to the subfamily of bacterial nucleotidyl cyclase toxins.

Activity of ExoY is strongly stimulated upon interaction with F-actin.

ExoY co-localises with F-actin in cells and alters F-actin turnover.

(1)

\section{Introduction}

Pseudomonas aeruginosa is a ubiquitous environmental bacterium and an opportunistic human pathogen. Equipped with different virulence factors and antibiotic resistance mechanisms, the bacteria colonise tissues and organs of both healthy and immunocompromised individuals and represent a major threat to patients with cystic fibrosis (for review (Engel and Balachandran, 2009; Hauser, 2009)). Because of its high viability in different environments or on various surfaces and its intrinsic resistance to many antibiotics, $P$. aeruginosa is a major cause of nosocomial infections, which are becoming a primary concern in hospitals all over the world (Fazeli et al., 2012).

During infection, clinical isolates of $P$. aeruginosa produce an extensive repertoire of virulence determinants including: (i) Exotoxin A, an ADP-ribosylating toxin secreted by the type 2 secretion system that enters eukaryotic cells by endocytosis where it inhibits elongation factor-2, thereby blocking polypeptide synthesis (for review (Michalska and Wolf, 2015)); (ii) Lipopolysaccharide, a major inflammatory molecule (Pier, 2007); (iii) Zinc metalloproteases such as ArpA, LasA and LasB that prevent flagellin-mediated immune recognition (Casilag et al., 2015); (iv) Phospholipases PlcH, PlcN, PldA, PldB that hydrolyse membrane components of the target cells (Barker et al., 2004; Jiang et al., 2014; Spencer and Brown, 2015); (v) ExlA, a recently characterised pore-forming toxin that causes host cell membrane disruption and is secreted by a two-partner secretion system by clinical P. aeruginosa isolates that lack a type 3 secretion system (T3SS) (Bouillot et al., 2017; Elsen et al., 2014); and (vi) several protein effectors, ExoS, ExoT, 
51 ExoU and ExoY, that are delivered to the host cells by a functional T3SS. The ExoS and 52 ExoT are toxins with an N-terminal GTPase-activating (GAP) domain and a C-terminal ADP-ribosyltransferase (ADPRT) domain (Iglewski et al., 1978; Riese et al., 2001). ExoS and ExoT are activated upon interaction with an eukaryotic factor (14-3-3 protein). Their N-terminal GAP-domains modulate the activity of small GTP-binding proteins of the Rho-family that control actin cytoskeleton organisation and rearrangements, while their C-terminal domains modify many proteins involved in vesicular trafficking, endocytosis, cell signaling or DNA synthesis regulation (Barbieri and Sun, 2004; Fu et al., 1993; Hauser, 2009). Therefore, ExoS and ExoT inhibit bacterial phagocytosis and facilitate $P$. aeruginosa movement across the epithelial monolayer (Goehring et al., 1999; Soong et al., 2008). Another T3SS effector, ExoU, belongs to the phospholipase A2 (PLA2) family (Sato et al., 2003; Sawa et al., 2014). This enzyme is activated upon interaction with ubiquitin or ubiquitinated proteins (Anderson et al., 2011; Tessmer et al., 2017) and hydrolyzes phospholipids and lysophospholipids (Sato et al., 2005; Tamura et al., 2004). The enzymatic activity of ExoU causes the disruption of cell membranes and cell death. As a result, this toxin is a major virulence factor responsible for acute lung injury in both animal models and patients with pneumonia caused by $P$. aeruginosa (Finck-Barbancon et al., 1997; Kurahashi et al., 1999).

The fourth described T3SS effector ExoY, a nucleotidyl cyclase toxin, was identified in 1998 (Yahr et al., 1998), by characterising P. aeruginosa transposon mutants defective in the T3SS apparatus. Several secreted proteins were absent in supernatant of these strains, including an unknown $\approx 42$-kDa polypeptide. Subsequent $\mathrm{N}$-terminal sequencing of this polypeptide and bioinformatic analysis identified the corresponding open reading frame in the P. aeruginosa genome database. Analysis of the amino acid sequence of this unknown protein showed homology with catalytic regions of the well-known 
calmodulin-activated adenylate cyclase toxins produced by Bordetella pertussis (CyaA or ACT) and Bacillus anthracis (edema factor, EF), the causative agents of whooping cough and anthrax, respectively (Carbonetti, 2010; Moayeri et al., 2015). Yahr et al. further cloned and expressed a recombinant ExoY and established that this toxin is endowed with an adenylate cyclase (AC) activity that was strongly stimulated by a heat-labile factor present in eukaryotic cell lysates. Yet, unlike CyaA and EF, the ExoY enzymatic activity was not stimulated by calmodulin (CaM) indicating that its activator was distinct from that of the other known AC bacterial toxins. The eukaryotic ExoY cofactor remained elusive until our group recently identified filamentous actin (F-actin) as the activator of ExoY (Belyy et al., 2016). Upon binding to F-actin ExoY undergoes structural rearrangements that increase its catalytic turnover rate to generate supraphysiological amounts of cyclic nucleotides, which in cell cultures disrupt cell signalling, inducing cytoskeleton disorganisation and eventually cell death (Sayner et al., 2004). Very recently, two groups independently demonstrated that ExoY activity can also lead to the inhibition of the host immune responses by suppressing the activation of TAK1 and decreasing the production of IL-1 $\beta$ (He et al., 2017; Jeon et al., 2017). The contribution of ExoY to P. aeruginosa virulence during acute and/or chronic infections were recently reviewed by T. Stevens and colleagues (Morrow et al., 2017). This mini-review summarizes some recent biochemical studies on ExoY and its regulation by actin.

\section{ExoY expression and secretion}

The exoY gene is present in almost $90 \%$ of clinical isolates (Feltman et al., 2001). In the reference PAO1 strain, the gene is not clustered with the ExoS and ExoT genes, and surrounded by two hypothetical genes of unknown function and not associated with an operon. A general T3SS transcriptional activator ExsA binds specifically to a -35 RNA 
101 polymerase binding site and an A-rich sequence upstream to the ExoY gene (Brutinel et 102 al., 2008). Translation of the corresponding mRNA results in a protein of 378 amino 103 acids and $41.7 \mathrm{kDa}$. Whether ExoY undergoes additional post-translational modifications 104 or associates with other proteins inside the bacteria remains unknown. Yet, by analogy 105 with other T3SS toxins, ExoY may need to interact with a presently unidentified 106 chaperone that could maintain the protein in a partially unfolded state until it is 107 delivered to the secretion apparatus (for review on T3SS chaperones (Burkinshaw and 108 Strynadka, 2014)).

\section{ExoY as a member of the family of bacterial nucleotidyl cyclase toxins}

111 Bacterial adenylate cyclases toxins are pathogenic factors able to invade eukaryotic cells

112 where they are stimulated by endogenous cofactors to produce large amounts of 3'5'113 cyclic nucleoside monophosphates (cNMP). This results in disruption of cell signalling 114 and alteration of cell physiology, eventually leading to cell death. Indeed, the well115 characterized CyaA toxin from B. pertussis and edema factor, EF, from B. anthracis are 116 key virulence factors of these organisms. They target mainly the immune cells to disable 117 the immune response and thus facilitate bacterial colonization of their hosts (for review 118 (Carbonetti, 2010; Moayeri et al., 2015)).

119 These two toxins are rather selective adenylate cyclase enzymes that only marginally 120 produce other cNMPs (Beckert et al., 2014). In contrast, $P$. aeruginosa ExoY is a 121 promiscuous nucleotidyl cyclase, which synthesizes cGMP, cAMP, cUMP and cCMP 122 (Bahre et al., 2015; Beckert et al., 2014).

123 The family of bacterial cyclase toxins can be divided into two subgroups based on the 124 nature of the activator. As discussed previously, while EF and CyaA are activated by 125 calmodulin, ExoY defines a novel sub-family of nucleotidyl cyclase toxins activated by 
126 actin: this latter also includes various ExoY-like (ExoY-L) modules identified in several 127 members of multifunctional autoprocessing RTX (MARTX) toxins that are produced by 128 various Gram negative pathogens (see below) (Belyy et al., 2016; Ziolo et al., 2014). The 129 overall amino acid sequence conservation between ExoY and EF or CyaA is rather low 130 (44.3\% and 40.9\% sequence similarity, respectively) (Belyy et al., 2016), but three 131 regions of these proteins display high similarity (up to 66\%). Site directed mutagenesis 132 and structural studies demonstrated that, in EF and CyaA, these three regions are 133 directly involved in substrate binding and catalysis (Drum et al., 2002; Glaser et al., 134 1989, 1991). The corresponding conserved amino acid sequences in ExoY are thus 135 predicted to be part of the catalytic site. Indeed, Yahr et al. originally showed that the 136 residues $\mathrm{K} 81$ and K88, in catalytic region 1 (CR1) of ExoY are essential for catalysis. By 137 analogy with EF and CyaA, these residues are likely to hold the $\alpha$-phosphate of the 138 bound nucleotide substrate. Residues D212 and D214 of the catalytic region 2 might 139 play a role in stabilising the $\beta$ - and $\gamma$-phosphates of the bound nucleotide via $\mathrm{Mg}^{2+}$. 140 Residues H291, D294 and N297 of the CR3 are conserved in CyaA and EF and were 141 shown to be involved in contacting the base moiety of the nucleotide (Drum et al., 2002; 142 Guo et al., 2005). By analogy with other T3SS substrates, the N-terminal residues of ExoY 143 are hypothesised to harbour a T3SS secretion signal and a potential chaperone-binding 144 domain (Maresso et al., 2006). The schematic structures of the toxins and partial 145 sequence alignments are presented in fig. 1. 
151 PaEXoY 70-VTTLIEEGFPTKGFSVKGKSSNWGPQAGFICVDQHLSKREDR-111

152 врСуаA 46-STSLIAEGVATKGLGVHAKSSDWGLQAGYIPVNPNLSKLFGR-88

153 BaEF 335-ATNLIKSGVATKGLNVHGKSSDWGPVAGYIPFDQDLSKKHGQ-376

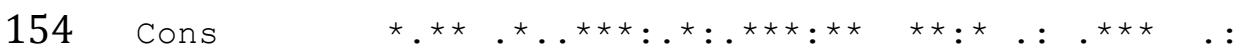

155

156 CR 2

157 PaEXOY 194-SEAVQVLASPACGL--AMTADYDLFLVAPSIE-223

158 BPCyaA 171-FEAVKVIGNAA-GI--PLTADIDMFAIMPHLS-199

159 BaEF 471-WRNIEVMAKNVEGVLKPLTADYDLFALAPSLT-502

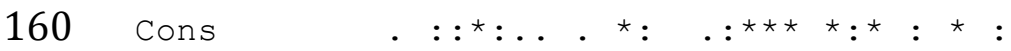

162 CR 3

163 PaEXoY 259-DMARGNITPRTRQLVDALNDCLGRGEH--REMFHHSDDAGNPGSHMGDN--305

164 BpCyaA 266-DMNIGVITDFELEVRNALNRRAHAVGA--QDVVQHGTEQNNP-FPEADEK-312

165 BaEF 543-DSTKGTLSNWQKQMLDRLNEAVKYTGYTGGDVVNHGTEQDNEEFPEKDNE-592

166 Cons

168 Figure 1. A. Schematic domain organisation of the catalytic domains of B. pertussis CyaA

169 (BpCyaA), B. anthracis EF (BaEF) and P. aeruginosa ExoY (PaExoY). The catalytic regions

170 CR1, CR2 and CR3 that display high sequence similarity are indicated by red boxes while

171 the CaM Binding Regions CBR of CyaA and EF are indicated by blue boxes. The Activator

172 Binding Region(s), ABR, of ExoY is/are still unknown. The predicted N-terminal T3SS

173 signal of P. aeruginosa ExoY (PaExoY) is indicated by an orange box.

174 B. Alignments of the catalytic regions CR1, CR2 and CR3. Bold amino acids are directly 175 involved in the catalytic process. The alignment was performed by the T-coffee 176 algorithm (Notredame et al., 2000).

\section{ExoY activation by filamentous actin}

179 Cyclic nucleotides are important messengers in intracellular signal transduction in 180 eukaryotes and bacteria. To avoid the detrimental influence of toxin activity in their 181 bacterial hosts, the nucleotidyl cyclase toxins possess a low basal activity in the 
182

183

184

185

186

187

188

189

prokaryotic environment and become active only after delivery to the eukaryotic cells where they are activated upon interaction with a specific activator. The activator of CyaA and EF has been known for a long time to be the highly conserved calciummediator calmodulin. In contrast, the activator of ExoY remained elusive until its recent identification as actin in our laboratory (Belyy et al., 2016), as briefly summerized below.

ExoY is toxic for numerous cell types, including mammalian and yeast cells (Arnoldo et al., 2008). This indicated that its activator should be evolutionary conserved in all eukaryotic cells. To identify the ExoY activator, a TAP-tagged inactivated variant of ExoY (to avoid cytotoxicity), ExoY-K81M, was expressed in yeast and affinity purified. The proteins that co-purified with the ExoY bait were analyzed by mass spectrometry. A label-free quantification score estimation of the data revealed that actin was the most abundant protein in the samples (apart the ExoY bait). This result suggested a specific interaction between ExoY and actin in yeast, and it was further confirmed, by cosedimentation and pull-down experiments, that in vitro the recombinant ExoY indeed associates with the polymeric form of mammalian actin. ExoY was further shown to bind along the lateral side of actin filaments. This lateral side binding inhibits the filament's disassembly and disturbs the binding and regulation of other lateral side binding proteins such as the Actin-depolymerizing factor (ADF). The lateral side binding of ExoY was thus shown to decrease the intrinsic or regulated turnover of actin filaments in vitro. In line with these findings, we showed that, in transfected cells, ExoY is recruited to actin filaments and increases the content of F-actin by stabilizing filaments and disturbing the balance of their assembly and disassembly by eukaryotic actin-binding proteins. Moreover, in vitro assays indicated that actin potently stimulates ExoY 
nucleotidyl cyclase activities in a dose-dependent manner with a half maximal activation

208 at about $0.2 \mu \mathrm{M}$ of ATP-Mg2+-loaded actin (Belyy et al., 2016), which correlates with the 209 critical concentration above which ATP-actin spontaneously polymerizes (i.e. $0.1 \mu \mathrm{M}$ 210 (Pollard et al., 2000))

211 Interestingly, certain toxins from the Multifunctional-Autoprocessing Repeats-in-ToXin 212 (MARTX) family produced by various pathogenic proteobacterial species, contain among 213 various effector domains an ExoY-like module. Ziolo et al. (Ziolo et al., 2014), indeed 214 demonstrated that the ExoY-like domain from the Vibrio vulnificus Biotype 3 MARTX 215 toxin is endowed with adenylate cyclase activity and is essential for virulence in mice. 216 We then showed that actin stimulates in a dose-dependent manner, a recombinant 217 ExoY-like module, VnExoY-L, derived from the MARTX toxin from Vibrio 218 nigripulchritudo, a marine pathogen infecting shrimps (Belyy et al., 2016). This suggests 219 that ExoY and the other ExoY-like modules present in MARTX toxins belong to a specific 220 subfamily of actin-activated nucleotidyl cyclase toxins. However, at variance with $P$. 221 aeruginosa ExoY, the ExoY-like modules from the V. vulnificus (Ziolo et al., 2014) or V. 222 nigripulchritudo (Belyy et al., 2016) MARTX toxins only synthesize cAMP. Hence, the 223 subfamily of actin-activated nucleotidyl cyclase toxins displays different substrate 224 selectivity, and may thus differ significantly in the range of cellular pathways they affect 225 in host cells.

226 By analogy with CyaA and EF, one can hypothesise, that upon interaction with the 227 activator, the catalytic regions CR1, 2, and 3 of ExoY are stabilised in an active 228 configuration, resulting in high catalytic activity. Indeed, the key step of the activation of 229 EF by CaM, is the stabilisation of a catalytic loop called switch B, corresponding to amino 230 acids H577-E592 of EF (Drum et al., 2002) and H298-K312 in CyaA (Guo et al., 2005). 231 The similar region CR3 in ExoY -residues H291-N305 - contains, an asparagine residue, 
232 N297, homologous to EF N583 or CyaA N304, which were shown to be important for

233 holding the ATP ribose in place upon substrate binding and hence the catalytic activity

234 of those enzymes.

235

5. Actin as a target and an activator of toxins

237

Actin is a $42-\mathrm{kDa}$ multifunctional protein, present either in a monomeric G-form (Gactin) or as a part of a filamentous linear polymer (F-actin). Actin is a highly conserved and essential component of every eukaryotic cell. As a matter of fact, the actin cytoskeleton is the target of a wide variety of toxins produced by various organisms

241 including many microbial pathogens (Colonne et al., 2016; Rappuoli and Montecucco, 242 1997). Several fungi and sponges produce nonproteinacous toxins, such as latrunculin, 243 cytochalasin, jasplakinolide, that target the actin cytoskeleton by modulating the G to F244 actin equilibrium in cells. Bacterial toxins are affecting the actin cytoskeleton either by 245 targeting specific actin-regulatory proteins or through direct modification of actin (for 246 review (Aktories et al., 2017)). For example, Clostridium difficile C2-toxin, C. perfringens 247 iota toxin and C. spiroforme toxin attack actin cytoskeleton by ADP-ribosylation of 248 Arg177 of actin. The modified actin acts as a capping protein and prevents further actin 249 elongation, poisoning actin polymerisation and leading to actin depolymerization in cells 250 (Perieteanu et al., 2010; Wegner and Aktories, 1988). By contrast, modification of actin 251 Thr148 by Photorhabdus luminescens TCC3 toxin leads to polymerisation and 252 aggregation of F-actin (Lang et al., 2017). Several MARTX toxins from Vibrio species and 253 other pathogens contain enzymatic domains that display an actin-crosslinking activity 254 that results in the formation of deleterious non-polymerisable actin oligomers that both 255 poison actin polymerisation and trap key regulatory proteins of the actin cytoskeleton 
dynamics such as formins in non-functional complexes (Fullner and Mekalanos, 2000;

257 Kudryashov et al., 2008).

258 Interestingly, apart from ExoY and ExoY-like toxins, another toxin has been reported to 259 be activated by actin. This toxin, YpkA (also known as Yop0) is a protein kinase 260 produced by various Yersinia species and delivered to target cells by a T3SS (Juris et al., 261 2000; Trasak et al., 2007). The YpkA/YopO kinase is activated upon binding to 262 monomeric actin and subsequent autophosphorylation. Recent structural and functional 263 studies revealed that YpkA binds to the subdomain 4 of actin (figure 2), and uses actin 264 as a bait to recruit actin-binding proteins (eg VASP, WASP, formin, and gelsolin) and 265 phosphorylate them (Lee et al., 2015). Phosphorylation of actin regulatory proteins by 266 YpkA/Yop0 appears as a novel regulatory mechanism by which pathogenic bacteria can 267 potentially manipulate actin dynamics, and thus impair phagocytosis (Ono, 2017; 268 Singaravelu et al., 2017).

\section{G-actin}

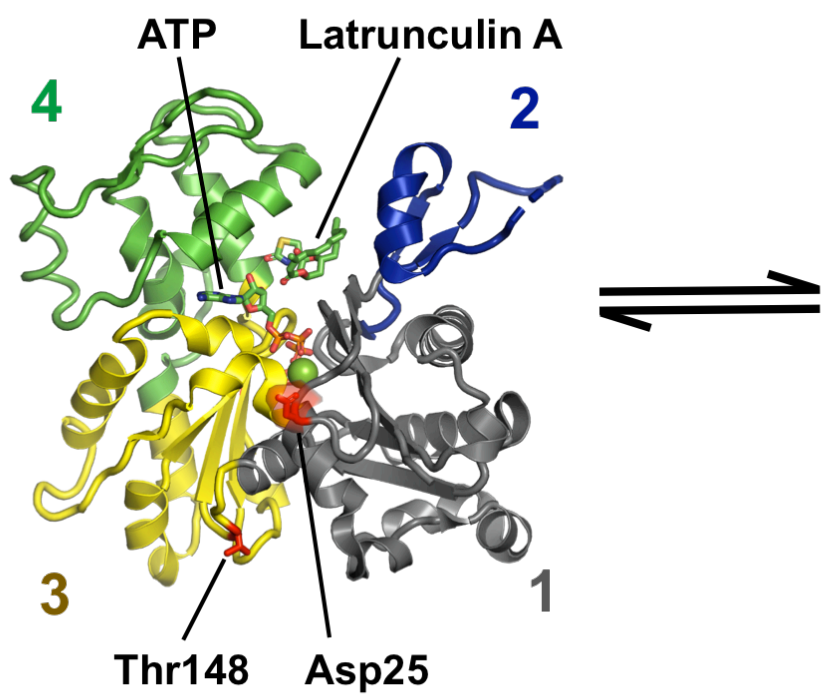

\section{F-actin}

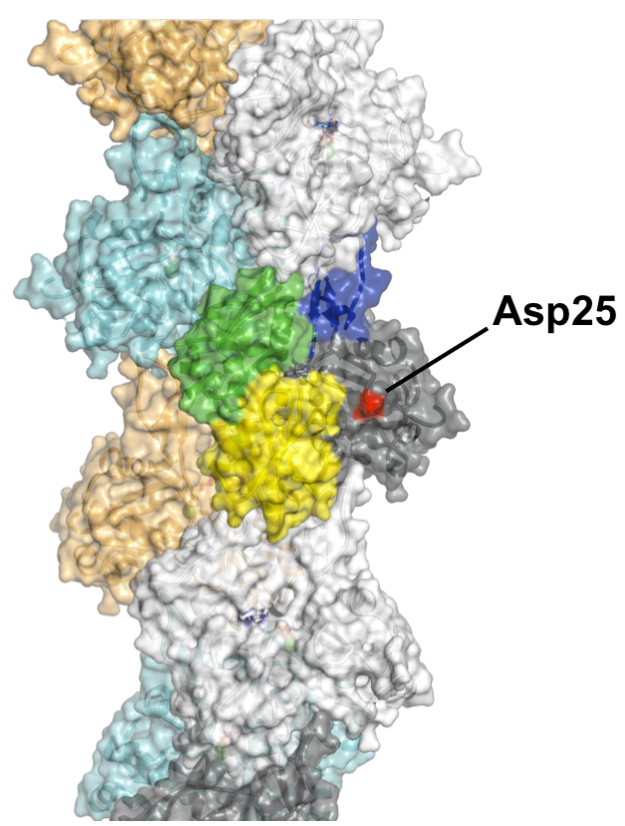

Figure 2 in JPG format 
272 Figure 2. Structures of G-actin bound to Latrunculin A (PDB 1IJJ), a toxin extracted from

273 the red sea sponge Latrunculia magnifica and inhibiting actin polymerization, and F274 actin (PDB 2ZWH). The 4 subdomains of G-actin are shown in different colours and 275 labeled from 1 to 4 . Actin subunits in F-actin are represented by their surface in 276 different colours, with one subunit shown with the same colour codes as G-actin. The 277 side chains of actin Thr148, which is modified by Photorhabdus luminescens TCC3 toxin, 278 and actin Asp25, whose mutation D25N abolishes the activation of ExoY by actin 279 filaments and its cytotoxicity in yeast, are shown in red ball-and-stick presentation. actin variants that do not activate ExoY

To further characterise the molecular determinants of the ExoY-actin interaction, we attempted to identify mutations in actin that prevent binding and activation of ExoY. For this, we performed a random mutagenesis of actin using as a model organism the yeast S. cerevisiae that contains a single actin-encoding gene ACT1 (figure 3). The actin gene was mutagenized in vitro by error-prone PCR (1) and cloned in a low-copy yeast plasmid 290 (carrying a His3 marker) $(2,3)$. The resulting library was then transformed into an engineered yeast strain (4), in which the chromosomal actin gene was inactivated while a functional wild-type copy of ACT1 gene was provided in trans by a plasmid bearing an uracil marker, URA3 (Belyy et al., 2015).

294 After transformation of the library, all transformed cells were pooled and replicated on a medium containing 5-fluoroorotic acid (5-FOA) to eliminate the plasmid carrying the uracil marker and the WT actin (5). The resulting cells thus relied only on the 297 mutagenized actin gene (on the His3-bearing plasmid) for growth and as actin is an essential protein, only cells harbouring functional variants of actin could survive this selection. The surviving yeast cells (i.e. containing mutagenized yet functional actins) 
were finally transformed with an ExoY-expressing plasmid and plated on a galactosecontaining medium in order to trigger ExoY expression (6). In these conditions only yeast carrying actin variants unable to activate ExoY could survive. Few surviving clones

303 were isolated and sequencing of their actin genes revealed two different alleles, both 304 with mutations of Asp25. Further experiments confirmed that this amino acid is indeed 305 crucial for ExoY activation: the single mutation D25N in actin was sufficient to render 306 yeast resistant to ExoY in vivo, and a corresponding yeast lysate was unable to activate 307 ExoY in in vitro assays. The D25 residue is well conserved among all isoforms of 308 eukaryotic actin. The acidic N-terminus of actin as well as the D24 and D25-residues 309 were shown to be an interaction site with myosin (Bertrand et al., 1988; von der Ecken 310 et al., 2016). This indicated that D25 is fully accessible at the surface of the actin filament 311 and could therefore establish direct contacts with ExoY. Our results also suggest that 312 ExoY might compete with myosin for binding to actin filaments, but such possible 313 competition and in vivo effects remain to be established.

314 In addition, the fact that the D25N mutation in actin is sufficient to confer resistance to 315 ExoY toxicity in yeast demonstrated that actin is the only activator of this toxin in this 316 organism. This observation was indeed crucial to ascertain that actin activates ExoY 317 through direct contacts and not indirectly via actin-binding proteins and/or actin post318 translational modifications (eg ubiquitinylation, (Terman and Kashina, 2013)). 


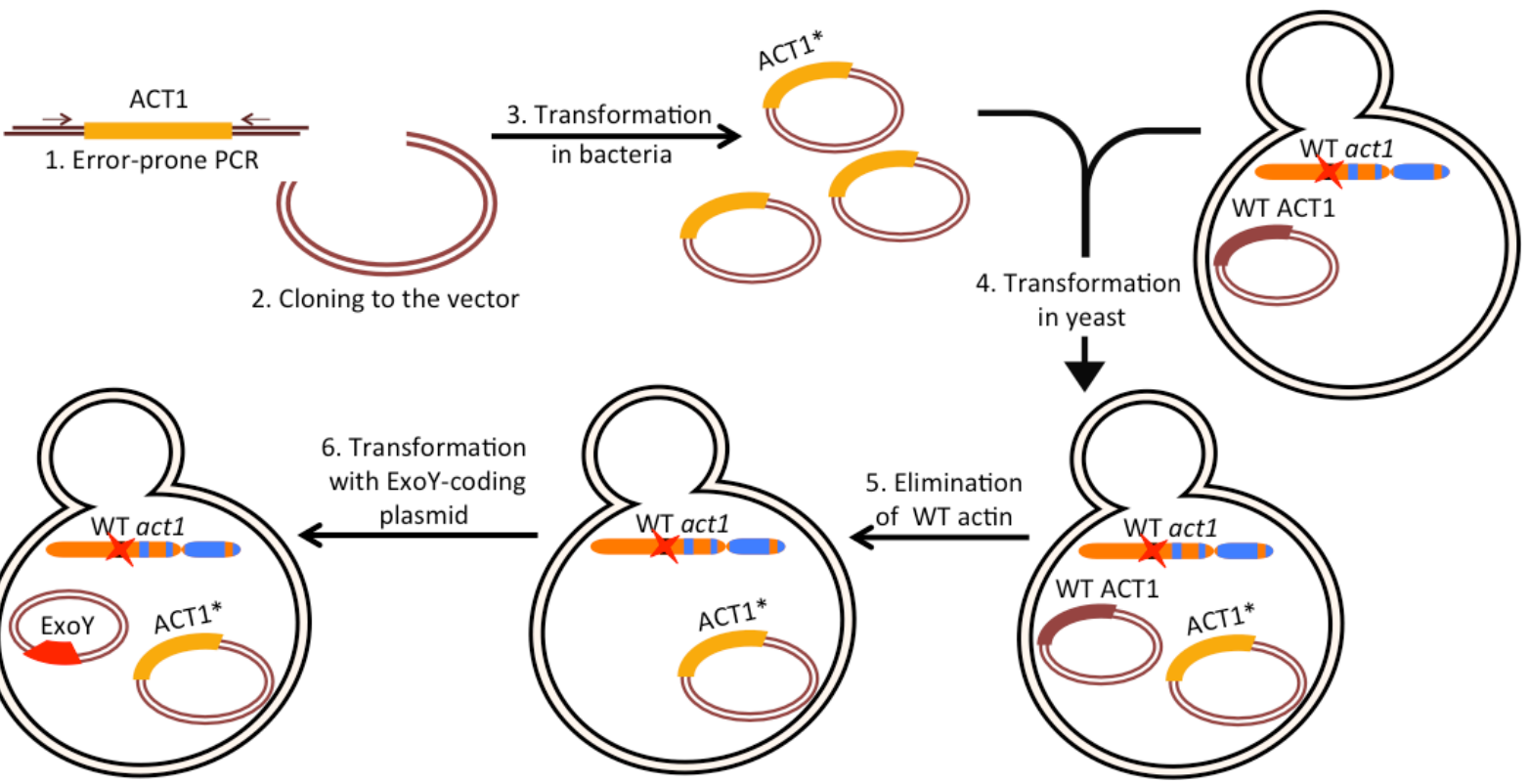

Figure 3 in JPG format

Figure 3. Strategy for actin mutagenesis and selection of ExoY-resistant actin variants in yeast. The key steps (1 to 6 ) are described in the main text.

7. Structural basis of ExoY discrimination between eukaryotic and homologous proteins of prokaryotic origin

The identification of actin as the ExoY activator also raises interesting questions from an evolutionary point of view. Indeed, bacteria possess several actin homologs (for review

331 (Shaevitz and Gitai, 2010)), such as MreB, FtsA or ParM. These proteins display 332 structural similarities to eukaryotic actin and are capable of ATP-dependent 333 polymerization into linear filaments. However as mentioned earlier, it is crucial that 334 ExoY (like other cyclase toxins) is kept inactive in its bacterial host to prevent any 335 toxicity. Therefore, this implies that the ExoY toxin must possess a high selectivity of 336 recognition of eukaryotic actins but not the endogenous $P$. aeruginosa actin-like 337 proteins. It is particularly noteworthy that the polypeptide region around the key Asp25 338 residue is organised in eukaryotic actins in a loop with a net negative charge, while it 
339 forms a turn with no net charge or a net positive charge between two beta-strands in 340 prokaryotic actin-like proteins (Figure 4). This structural difference may be a key

341 feature allowing ExoY to specifically discriminate between the eukaryotic actins and the 342 prokaryotic actin homologs.
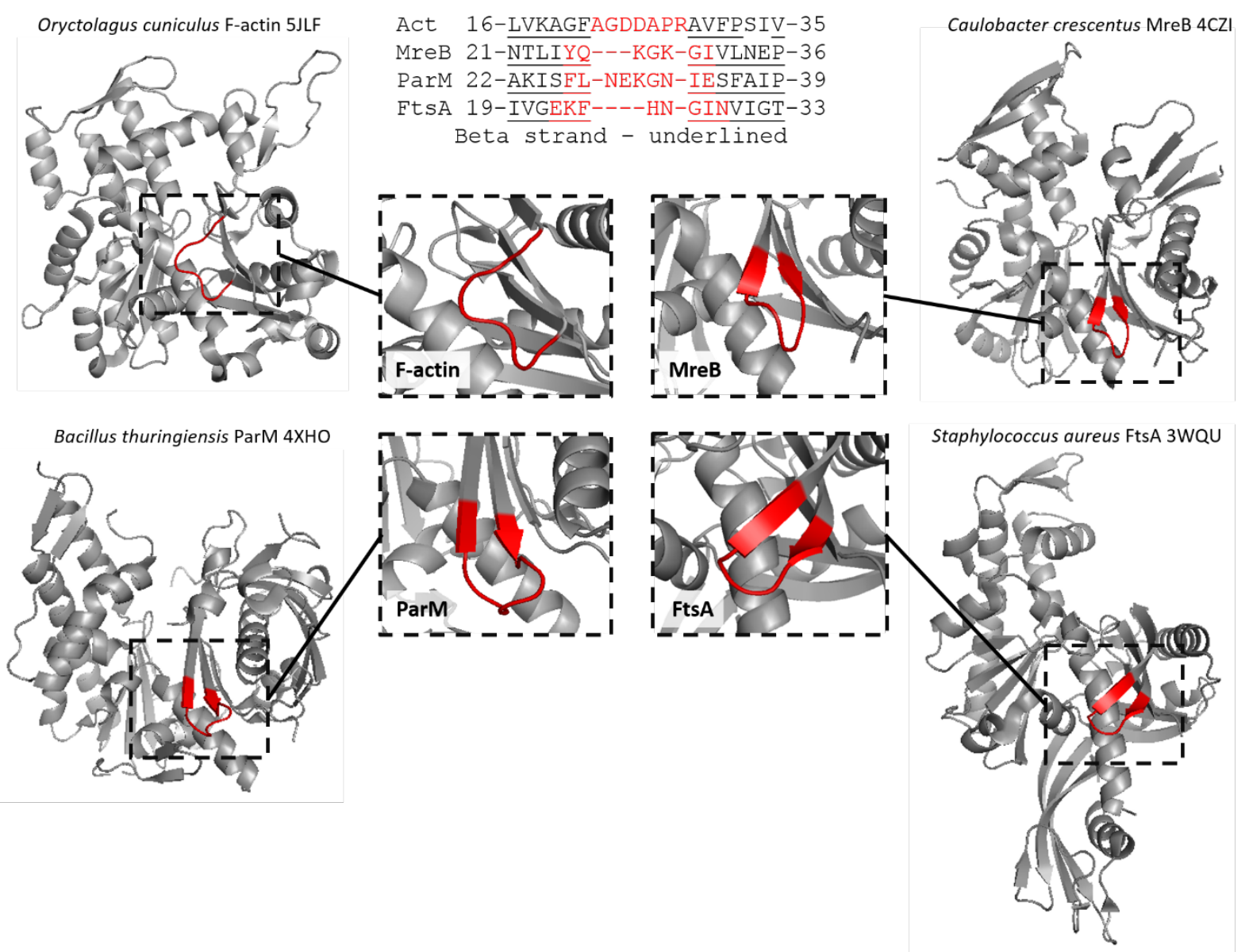

Figure 4 in JPG format

Figure 4. Comparison of the structural models of an actin monomer from rabbit muscle

348 (5JLF, (von der Ecken et al., 2016)), Caulobacter crescentus MreB (4CZI, (van den Ent et al., 2014)), Bacillus thuringiensis ParM (4XHO, Jiang et al., unpublished) and 
highlighted in red. In the sequence alignment panel above, the amino acids belonging to the flanking beta-strands are underlined.

\section{Conclusions}

Bacterial nucleotidyl cyclase toxins are potent virulence factors that hijack cell cNMP signalling in eukaryotic cells. These toxins are inactive inside their bacterial host and need to interact with specific eukaryotic cofactors in eukaryotic target cells to convey their high catalytic activity. The recent discovery of actin as the activator of ExoY and ExoY-like modules present in MARTX toxins suggests that the family of bacterial nucleotidyl cyclase toxins is composed of two distinct branches distinguished by the nature of their activator, calmodulin vs actin. It is noteworthy that both activators are abundant proteins and highly conserved in the entire eukaryotic kingdom. The recent advances in understanding of the mechanism of ExoY activation are now paving the way for further exploration of the biological function of this virulence factor that still remains the least characterized protein among the four T3SS effectors of P. aeruginosa. Among key issues that are currently of prime interest are (i) the structural basis for ExoY activation by F-actin and its broad nucleotide specificity; (ii) the potential influence of the P. aeruginosa exotoxins ExoS/ExoT, that modulate the actin cytoskeleton dynamics, on ExoY activation in vivo; (iii) the characterisation of putative ExoY chaperones in the bacterial host and (iv) the characterisation of the functional and enzymatic specificities of ExoY-like toxins from other gram-negative pathogens.

\section{Acknowledgements}

The authors thank Jenny Lee Thomassin for the critical reading of the manuscript. A.B. was supported by a stipend from the Pasteur-Paris University (PPU) International PhD Program. The authors were funded by CNRS and by the PTR program of the Institut 
Pasteur (PTR425 and PTR43-16). The authors declare no competing financial interest.

The funding sources had no involvement in the preparation of the article.

\section{References}

Aktories, K., Schwan, C., Lang, A.E., 2017. ADP-Ribosylation and Cross-Linking of Actin by Bacterial Protein Toxins. Handb. Exp. Pharmacol. 235, 179-206. https://doi.org/10.1007/164_2016_26

Anderson, D.M., Schmalzer, K.M., Sato, H., Casey, M., Terhune, S.S., Haas, A.L., Feix, J.B., Frank, D.W., 2011. Ubiquitin and ubiquitin-modified proteins activate the Pseudomonas aeruginosa T3SS cytotoxin, ExoU. Mol. Microbiol. 82, 1454-1467. https://doi.org/10.1111/j.1365-2958.2011.07904.x

Arnoldo, A., Curak, J., Kittanakom, S., Chevelev, I., Lee, V.T., Sahebol-Amri, M., Koscik, B., Ljuma, L., Roy, P.J., Bedalov, A., Giaever, G., Nislow, C., Merrill, A.R., Lory, S., Stagljar, I., 2008. Identification of small molecule inhibitors of Pseudomonas aeruginosa exoenzyme $S$ using a yeast phenotypic screen. PLoS Genet. 4, e1000005. https://doi.org/10.1371/journal.pgen.1000005

Bahre, H., Hartwig, C., Munder, A., Wolter, S., Stelzer, T., Schirmer, B., Beckert, U., Frank, D.W., Tummler, B., Kaever, V., Seifert, R., 2015. cCMP and cUMP occur in vivo. Biochem. Biophys. Res. Commun. 460, 909-914. https://doi.org/10.1016/j.bbrc.2015.03.115

Barbieri, J.T., Sun, J., 2004. Pseudomonas aeruginosa ExoS and ExoT. Rev. Physiol. Biochem. Pharmacol. 152, 79-92. https://doi.org/10.1007/s10254-004-0031-7

Barker, A.P., Vasil, A.I., Filloux, A., Ball, G., Wilderman, P.J., Vasil, M.L., 2004. A novel extracellular phospholipase $C$ of Pseudomonas aeruginosa is required for phospholipid chemotaxis. Mol. Microbiol. 53, 1089-1098. https://doi.org/10.1111/j.1365-2958.2004.04189.x

Beckert, U., Wolter, S., Hartwig, C., Bahre, H., Kaever, V., Ladant, D., Frank, D.W., Seifert, R., 2014. ExoY from Pseudomonas aeruginosa is a nucleotidyl cyclase with preference for cGMP and cUMP formation. Biochem. Biophys. Res. Commun. 450, 870-874. https://doi.org/10.1016/j.bbrc.2014.06.088

Belyy, A., Raoux-Barbot, D., Saveanu, C., Namane, A., Ogryzko, V., Worpenberg, L., David, V., Henriot, V., Fellous, S., Merrifield, C., Assayag, E., Ladant, D., Renault, L., Mechold, U., 2016. Actin activates Pseudomonas aeruginosa ExoY nucleotidyl cyclase toxin and ExoY-like effector domains from MARTX toxins. Nat. Commun. 7, 13582. https://doi.org/10.1038/ncomms13582

Belyy, A., Tabakova, I., Lang, A.E., Jank, T., Belyi, Y., Aktories, K., 2015. Roles of Asp179 and Glu270 in ADP-Ribosylation of Actin by Clostridium perfringens Iota Toxin. PloS One 10, e0145708. https://doi.org/10.1371/journal.pone.0145708

Bertrand, R., Chaussepied, P., Kassab, R., Boyer, M., Roustan, C., Benyamin, Y., 1988. Cross-linking of the skeletal myosin subfragment 1 heavy chain to the $\mathrm{N}$-terminal actin segment of residues 40-113. Biochemistry (Mosc.) 27, 5728-5736.

Bouillot, S., Munro, P., Gallet, B., Reboud, E., Cretin, F., Golovkine, G., Schoehn, G., Attree, I., Lemichez, E., Huber, P., 2017. Pseudomonas aeruginosa Exolysin promotes bacterial growth in lungs, alveolar damage and bacterial dissemination. Sci. Rep. 7, 2120. https://doi.org/10.1038/s41598-017-02349-0

Brutinel, E.D., Vakulskas, C.A., Brady, K.M., Yahr, T.L., 2008. Characterization of ExsA and of ExsA-dependent promoters required for expression of the Pseudomonas 
aeruginosa type III secretion system. Mol. Microbiol. 68, 657-671. https://doi.org/10.1111/j.1365-2958.2008.06179.x

Burkinshaw, B.J., Strynadka, N.C.J., 2014. Assembly and structure of the T3SS. Biochim. Biophys. Acta 1843, 1649-1663. https://doi.org/10.1016/j.bbamcr.2014.01.035 Carbonetti, N.H., 2010. Pertussis toxin and adenylate cyclase toxin: key virulence factors of Bordetella pertussis and cell biology tools. Future Microbiol. 5, 455-469. https://doi.org/10.2217/fmb.09.133

Casilag, F., Lorenz, A., Krueger, J., Klawonn, F., Weiss, S., Haussler, S., 2015. The LasB Elastase of Pseudomonas aeruginosa Acts in Concert with Alkaline Protease AprA To Prevent Flagellin-Mediated Immune Recognition. Infect. Immun. 84, 162-171. https://doi.org/10.1128/IAI.00939-15

Colonne, P.M., Winchell, C.G., Voth, D.E., 2016. Hijacking Host Cell Highways: Manipulation of the Host Actin Cytoskeleton by Obligate Intracellular Bacterial Pathogens. Front. Cell. Infect. Microbiol. 6, 107. https://doi.org/10.3389/fcimb.2016.00107

Drum, C.L., Yan, S.-Z., Bard, J., Shen, Y.-Q., Lu, D., Soelaiman, S., Grabarek, Z., Bohm, A., Tang, W.-J., 2002. Structural basis for the activation of anthrax adenylyl cyclase exotoxin by calmodulin. Nature 415, 396-402. https://doi.org/10.1038/415396a

Elsen, S., Huber, P., Bouillot, S., Coute, Y., Fournier, P., Dubois, Y., Timsit, J.-F., Maurin, M., Attree, I., 2014. A type III secretion negative clinical strain of Pseudomonas aeruginosa employs a two-partner secreted exolysin to induce hemorrhagic pneumonia. Cell Host Microbe 15, 164-176. https://doi.org/10.1016/j.chom.2014.01.003

Engel, J., Balachandran, P., 2009. Role of Pseudomonas aeruginosa type III effectors in disease. Curr. Opin. Microbiol. 12, 61-66. https://doi.org/10.1016/j.mib.2008.12.007

Fazeli, H., Akbari, R., Moghim, S., Narimani, T., Arabestani, M.R., Ghoddousi, A.R., 2012. Pseudomonas aeruginosa infections in patients, hospital means, and personnel's specimens. J. Res. Med. Sci. Off. J. Isfahan Univ. Med. Sci. 17, 332-337.

Feltman, H., Schulert, G., Khan, S., Jain, M., Peterson, L., Hauser, A.R., 2001. Prevalence of type III secretion genes in clinical and environmental isolates of Pseudomonas aeruginosa. Microbiol. Read. Engl. 147, 2659-2669. https://doi.org/10.1099/00221287-147-10-2659

Finck-Barbancon, V., Goranson, J., Zhu, L., Sawa, T., Wiener-Kronish, J.P., Fleiszig, S.M., Wu, C., Mende-Mueller, L., Frank, D.W., 1997. ExoU expression by Pseudomonas aeruginosa correlates with acute cytotoxicity and epithelial injury. Mol. Microbiol. 25, 547-557.

Fu, H., Coburn, J., Collier, R.J., 1993. The eukaryotic host factor that activates exoenzyme $\mathrm{S}$ of Pseudomonas aeruginosa is a member of the 14-3-3 protein family. Proc. Natl. Acad. Sci. U. S. A. 90, 2320-2324.

Fujita, J., Maeda, Y., Nagao, C., Tsuchiya, Y., Miyazaki, Y., Hirose, M., Mizohata, E., Matsumoto, Y., Inoue, T., Mizuguchi, K., Matsumura, H., 2014. Crystal structure of FtsA from Staphylococcus aureus. FEBS Lett. 588, 1879-1885. https://doi.org/10.1016/j.febslet.2014.04.008

Fullner, K.J., Mekalanos, J.J., 2000. In vivo covalent cross-linking of cellular actin by the Vibrio cholerae RTX toxin. EMBO J. 19, 5315-5323. https://doi.org/10.1093/emboj/19.20.5315

Glaser, P., Elmaoglou-Lazaridou, A., Krin, E., Ladant, D., Barzu, O., Danchin, A., 1989. Identification of residues essential for catalysis and binding of calmodulin in 
Bordetella pertussis adenylate cyclase by site-directed mutagenesis. EMBO J. 8, 967-972.

Glaser, P., Munier, H., Gilles, A.M., Krin, E., Porumb, T., Barzu, O., Sarfati, R., Pellecuer, C., Danchin, A., 1991. Functional consequences of single amino acid substitutions in calmodulin-activated adenylate cyclase of Bordetella pertussis. EMBO J. 10, 1683-1688.

Goehring, U.M., Schmidt, G., Pederson, K.J., Aktories, K., Barbieri, J.T., 1999. The Nterminal domain of Pseudomonas aeruginosa exoenzyme $\mathrm{S}$ is a GTPase-activating protein for Rho GTPases. J. Biol. Chem. 274, 36369-36372.

Guo, Q., Shen, Y., Lee, Y.-S., Gibbs, C.S., Mrksich, M., Tang, W.-J., 2005. Structural basis for the interaction of Bordetella pertussis adenylyl cyclase toxin with calmodulin. EMBO J. 24, 3190-3201. https://doi.org/10.1038/sj.emboj.7600800

Hauser, A.R., 2009. The type III secretion system of Pseudomonas aeruginosa: infection by injection. Nat. Rev. Microbiol. 7, 654-665. https://doi.org/10.1038/nrmicro2199

He, C., Zhou, Y., Liu, F., Liu, H., Tan, H., Jin, S., Wu, W., Ge, B., 2017. Bacterial Nucleotidyl Cyclase Inhibits the Host Innate Immune Response by Suppressing TAK1 Activation. Infect. Immun. 85. https://doi.org/10.1128/IAI.00239-17

Iglewski, B.H., Sadoff, J., Bjorn, M.J., Maxwell, E.S., 1978. Pseudomonas aeruginosa exoenzyme S: an adenosine diphosphate ribosyltransferase distinct from toxin A. Proc. Natl. Acad. Sci. U. S. A. 75, 3211-3215.

Jeon, J., Kim, Y.-J., Shin, H., Ha, U.-H., 2017. T3SS effector ExoY reduces inflammasomerelated responses by suppressing bacterial motility and delaying activation of NF-kappaB and caspase-1. FEBS J. https://doi.org/10.1111/febs.14199

Jiang, F., Waterfield, N.R., Yang, J., Yang, G., Jin, Q., 2014. A Pseudomonas aeruginosa type VI secretion phospholipase D effector targets both prokaryotic and eukaryotic cells. Cell Host Microbe 15, 600-610. https://doi.org/10.1016/j.chom.2014.04.010

Juris, S.J., Rudolph, A.E., Huddler, D., Orth, K., Dixon, J.E., 2000. A distinctive role for the Yersinia protein kinase: actin binding, kinase activation, and cytoskeleton disruption. Proc. Natl. Acad. Sci. U. S. A. 97, 9431-9436. https://doi.org/10.1073/pnas.170281997

Kudryashov, D.S., Durer, Z.A.O., Ytterberg, A.J., Sawaya, M.R., Pashkov, I., Prochazkova, K., Yeates, T.O., Loo, R.R.O., Loo, J.A., Satchell, K.J.F., Reisler, E., 2008. Connecting actin monomers by iso-peptide bond is a toxicity mechanism of the Vibrio cholerae MARTX toxin. Proc. Natl. Acad. Sci. U. S. A. 105, 18537-18542. https://doi.org/10.1073/pnas.0808082105

Kurahashi, K., Kajikawa, O., Sawa, T., Ohara, M., Gropper, M.A., Frank, D.W., Martin, T.R., Wiener-Kronish, J.P., 1999. Pathogenesis of septic shock in Pseudomonas aeruginosa pneumonia. J. Clin. Invest. 104, 743-750. https://doi.org/10.1172/JCI7124

Lang, A.E., Qu, Z., Schwan, C., Silvan, U., Unger, A., Schoenenberger, C.-A., Aktories, K., Mannherz, H.G., 2017. Actin ADP-ribosylation at Threonine148 by Photorhabdus luminescens toxin TccC3 induces aggregation of intracellular F-actin. Cell. Microbiol. 19. https://doi.org/10.1111/cmi.12636

Lee, W.L., Grimes, J.M., Robinson, R.C., 2015. Yersinia effector Yop0 uses actin as bait to phosphorylate proteins that regulate actin polymerization. Nat. Struct. Mol. Biol. 22, 248-255. https://doi.org/10.1038/nsmb.2964

Maresso, A.W., Frank, D.W., Barbieri, J.T., 2006. CHAPTER 14 - Pseudomonas aeruginosa 
toxins, in: The Comprehensive Sourcebook of Bacterial Protein Toxins (Third Edition). Academic Press, London, pp. 257-269. https://doi.org/10.1016/B978012088445-2/50019-6

Michalska, M., Wolf, P., 2015. Pseudomonas Exotoxin A: optimized by evolution for effective killing. Front. Microbiol. 6, 963. https://doi.org/10.3389/fmicb.2015.00963

Moayeri, M., Leppla, S.H., Vrentas, C., Pomerantsev, A.P., Liu, S., 2015. Anthrax Pathogenesis. Annu. Rev. Microbiol. 69, 185-208. https://doi.org/10.1146/annurev-micro-091014-104523

Morrow, K.A., Frank, D.W., Balczon, R., Stevens, T., 2017. The Pseudomonas aeruginosa Exoenzyme Y: A Promiscuous Nucleotidyl Cyclase Edema Factor and Virulence Determinant. Handb. Exp. Pharmacol. 238, 67-85. https://doi.org/10.1007/164_2016_5003

Notredame, C., Higgins, D.G., Heringa, J., 2000. T-Coffee: A novel method for fast and accurate multiple sequence alignment. J. Mol. Biol. 302, 205-217. https://doi.org/10.1006/jmbi.2000.4042

Ono, S., 2017. A plague of actin disassembly. J. Biol. Chem. 292, 8101-8102. https://doi.org/10.1074/jbc.H116.757971

Perieteanu, A.A., Visschedyk, D.D., Merrill, A.R., Dawson, J.F., 2010. ADP-ribosylation of cross-linked actin generates barbed-end polymerization-deficient F-actin oligomers. Biochemistry (Mosc.) 49, 8944-8954. https://doi.org/10.1021/bi1008062

Pier, G.B., 2007. Pseudomonas aeruginosa lipopolysaccharide: a major virulence factor, initiator of inflammation and target for effective immunity. Int. J. Med. Microbiol. IJMM 297, 277-295. https://doi.org/10.1016/j.ijmm.2007.03.012

Pollard, T.D., Blanchoin, L., Mullins, R.D., 2000. Molecular mechanisms controlling actin filament dynamics in nonmuscle cells. Annu. Rev. Biophys. Biomol. Struct. 29, 545-576. https://doi.org/10.1146/annurev.biophys.29.1.545

Rappuoli, R., Montecucco, C., 1997. Guidebook to Protein Toxins and Their Use in Cell Biology. OUP/Sambrook and Tooze Publications.

Riese, M.J., Wittinghofer, A., Barbieri, J.T., 2001. ADP ribosylation of Arg41 of Rap by ExoS inhibits the ability of Rap to interact with its guanine nucleotide exchange factor, C3G. Biochemistry (Mosc.) 40, 3289-3294.

Sato, H., Feix, J.B., Hillard, C.J., Frank, D.W., 2005. Characterization of phospholipase activity of the Pseudomonas aeruginosa type III cytotoxin, ExoU. J. Bacteriol. 187, 1192-1195. https://doi.org/10.1128/JB.187.3.1192-1195.2005

Sato, H., Frank, D.W., Hillard, C.J., Feix, J.B., Pankhaniya, R.R., Moriyama, K., FinckBarbancon, V., Buchaklian, A., Lei, M., Long, R.M., Wiener-Kronish, J., Sawa, T., 2003. The mechanism of action of the Pseudomonas aeruginosa-encoded type III cytotoxin, ExoU. EMBO J. 22, 2959-2969. https://doi.org/10.1093/emboj/cdg290

Sawa, T., Shimizu, M., Moriyama, K., Wiener-Kronish, J.P., 2014. Association between Pseudomonas aeruginosa type III secretion, antibiotic resistance, and clinical outcome: a review. Crit. Care Lond. Engl. 18, 668. https://doi.org/10.1186/s13054-014-0668-9

Sayner, S.L., Frank, D.W., King, J., Chen, H., VandeWaa, J., Stevens, T., 2004. Paradoxical cAMP-induced lung endothelial hyperpermeability revealed by Pseudomonas aeruginosa ExoY. Circ. Res. 95, 196-203. https://doi.org/10.1161/01.RES.0000134922.25721.d9 
Shaevitz, J.W., Gitai, Z., 2010. The structure and function of bacterial actin homologs. Cold Spring Harb. Perspect. Biol. 2, a000364. https://doi.org/10.1101/cshperspect.a000364

Singaravelu, P., Lee, W.L., Wee, S., Ghoshdastider, U., Ding, K., Gunaratne, J., Grimes, J.M., Swaminathan, K., Robinson, R.C., 2017. Yersinia effector protein (Yop0)-mediated phosphorylation of host gelsolin causes calcium-independent activation leading to disruption of actin dynamics. J. Biol. Chem. 292, 8092-8100. https://doi.org/10.1074/jbc.M116.757971

Soong, G., Parker, D., Magargee, M., Prince, A.S., 2008. The type III toxins of Pseudomonas aeruginosa disrupt epithelial barrier function. J. Bacteriol. 190, 2814-2821. https://doi.org/10.1128/JB.01567-07

Spencer, C., Brown, H.A., 2015. Biochemical characterization of a Pseudomonas aeruginosa phospholipase D. Biochemistry (Mosc.) 54, 1208-1218. https://doi.org/10.1021/bi501291t

Tamura, M., Ajayi, T., Allmond, L.R., Moriyama, K., Wiener-Kronish, J.P., Sawa, T., 2004. Lysophospholipase A activity of Pseudomonas aeruginosa type III secretory toxin ExoU. Biochem. Biophys. Res. Commun. 316, 323-331. https://doi.org/10.1016/j.bbrc.2004.02.050

Terman, J.R., Kashina, A., 2013. Post-translational modification and regulation of actin. Curr. Opin. Cell Biol. 25, 30-38. https://doi.org/10.1016/j.ceb.2012.10.009

Tessmer, M.H., Anderson, D.M., Buchaklian, A., Frank, D.W., Feix, J.B., 2017. Cooperative Substrate-Cofactor Interactions and Membrane Localization of the Bacterial Phospholipase A2 (PLA2) Enzyme, ExoU. J. Biol. Chem. 292, 3411-3419. https://doi.org/10.1074/jbc.M116.760074

Trasak, C., Zenner, G., Vogel, A., Yuksekdag, G., Rost, R., Haase, I., Fischer, M., Israel, L., Imhof, A., Linder, S., Schleicher, M., Aepfelbacher, M., 2007. Yersinia protein kinase YopO is activated by a novel G-actin binding process. J. Biol. Chem. 282, 2268-2277. https://doi.org/10.1074/jbc.M610071200

van den Ent, F., Izore, T., Bharat, T.A., Johnson, C.M., Lowe, J., 2014. Bacterial actin MreB forms antiparallel double filaments. eLife 3, e02634. https://doi.org/10.7554/eLife.02634

von der Ecken, J., Heissler, S.M., Pathan-Chhatbar, S., Manstein, D.J., Raunser, S., 2016. Cryo-EM structure of a human cytoplasmic actomyosin complex at near-atomic resolution. Nature 534, 724-728. https://doi.org/10.1038/nature18295

Wegner, A., Aktories, K., 1988. ADP-ribosylated actin caps the barbed ends of actin filaments. J. Biol. Chem. 263, 13739-13742.

Yahr, T.L., Vallis, A.J., Hancock, M.K., Barbieri, J.T., Frank, D.W., 1998. ExoY, an adenylate cyclase secreted by the Pseudomonas aeruginosa type III system. Proc. Natl. Acad. Sci. U. S. A. 95, 13899-13904.

Ziolo, K.J., Jeong, H.-G., Kwak, J.S., Yang, S., Lavker, R.M., Satchell, K.J.F., 2014. Vibrio vulnificus biotype 3 multifunctional autoprocessing RTX toxin is an adenylate cyclase toxin essential for virulence in mice. Infect. Immun. 82, 2148-2157. https://doi.org/10.1128/IAI.00017-14 